\title{
Different effects of scopolamine on learning, memory, and nitric oxide metabolite levels in hippocampal tissues of ovariectomized and Sham-operated rats
}

Diferentes efeitos da escopolamina no aprendizado, na memória e nos níveis dos metabólitos do óxido nítrico no tecido hipocampal de ratas ooforectomizadas e controles operadas sem ooforectomia

Hamid Azizi-Malekabadi', Mahmoud Hosseini², Mohammad Soukhtanloo³, Reihaneh Sadeghian4, Masoud Fereidoni4 Fatimeh Khodabandehloo ${ }^{5}$

\begin{abstract}
Different effects of scopolamine on learning, memory, and nitric oxide (NO) metabolites in hippocampal tissues of ovariectomized (OVX) and sham-operated rats were investigated. The animals in the Sham-Scopolamine (Sham-Sco) and OVX-Scopolamine (OVX-Sco) Groups were treated with $2 \mathrm{mg} / \mathrm{kg}$ scopolamine before undergoing the Morris water maze, while the animals in the Sham and OVX Groups received saline. The time latency and path length were significantly higher in both the Sham-Sco and the OVX-Sco Groups, in comparison with the Sham and OVX Groups, respectively ( $p<0.001)$. Significantly lower NO metabolite levels in the hippocampi of the Sham-Sco Group were observed, compared with the Sham Group ( $p<0.001)$, while there was no significant difference between the OVX-Sco and OVX Groups. The decreased NO level in the hippocampus may play a role in the learning and memory deficits induced by scopolamine. However, it seems that the effect of scopolamine on hippocampal NO differs between situations of presence and absence of ovarian hormones.
\end{abstract}

Key words: Morris water maze, scopolamine, ovariectomized, rat, hippocampus, nitric oxide.

\section{RESUMO}

Diferentes efeitos da escopolamina no aprendizado, na memória e nos níveis dos metabólitos do óxido nítrico (ON) no tecido hipocampal de ratas ovariectomizadas $(\mathrm{OVX})$ e controles com cirurgia sem ooforectomia (Grupo Sham) foram investigados. Os animais dos grupos Sham-Escopolamina (Sham-Sco) e OVX-Escopolamina (OVX-Sco) foram tratados com escopolamina $2 \mathrm{mg} / \mathrm{kg}$ antes de entrar no labirinto aquático de Morris, enquanto aqueles dos grupos Sham e OVX receberam solução salina. A latência de tempo e o comprimento do caminho foram significativamente maiores nos Grupos Sham-Sco e OVX-Sco em comparação com os grupos Sham e OVX, respectivamente $(p<0,001)$. Foram observados níveis significativamente mais baixos de metabólitos do ON nos hipocampos do Grupo Sham-Sco em comparação aos niveis do Sham ( $p<0,001$ ), enquanto não foi observada diferença significativa entre os Grupos OVX-Sco e OVX. A diminuição do nível de ON no hipocampo pode ter um papel no aprendizado e nos déficits de memória induzidos pela escopolamina. No entanto, parece que este efeito da escopolamina no ON hipocampal é diferente em situações de presença ou ausência de hormônios ovarianos.

Palavras-Chave: labirinto aquático de Morris, escopolamina, ooforectomia, rato, hipocampo, óxido nítrico.

\footnotetext{
'Faculty of Basic Sciences, Islamic Azad University, Khorasgan Branch, Isfahan, Iran;

${ }^{2}$ Neuroscience Research Center and Department of Physiology, Mashhad University of Medical Sciences, Mashhad, Iran;

${ }^{3}$ Department of Biochemistry, School of Medicine, Mashhad University of Medical Sciences, Mashhad, Iran;

${ }^{4}$ Deptartment of Biology, Faculty of Sciences, Ferdowsi University of Mashhad, Mashhad, Iran;

${ }^{5}$ Department of Physiology, School of Medicine, AJA University of Medical Sciences, Tehran, Iran.

Correspondence: Mahmoud Hosseini; Deptartemnt of Physiology, School of Medicine, Mashhad University of Medical Sciences; Mashhad - Iran;

E-mail:hosseinim@MUMS.ac.ir
}

Support: Vice-presidency of Research of Islamic Azad University, Khorasgan Branch, Isfahan.

Conflict of interest: There is no conflict of interest to declare.

Received 21 August 2011; Received in final form 30 January 2012; Accepted 07 February 2012 
There is evidence that the change in the levels of steroid hormones, particularly estradiol, has a critical role in senile cognitive impairment. The source of sex hormones, which reach the brain, is the gonads. However, estrogens and androgens are synthesized in brain areas such as the hippocampus and they modulate the function of the hippocampus as an important center, which is involved in learning and memory ${ }^{1,2}$.

The positive effect ${ }^{3}$, no effect $t^{4,5}$, or even negative effect ${ }^{6}$ of estrogen on learning and memory has been reported. The interaction of sex hormones with the neurotransmitter systems such as glutamatergic, serotonergic, adrenergic, dopaminergic, and cholinergic, which are involved in cognition, memory and learning, has been reported ${ }^{7-11}$.

The central cholinergic system is the most important neurotransmitter system, which is involved in several aspects of cognition and memory, and the cholinergic basis of memory dysfunction and cognitive decline in diseases such as Alzheimer's has also been well-documented ${ }^{12,13}$. The interaction of estrogen with the central cholinergic system has also been suggested. On one hand, estrogen has been reported to stimulate choline acetyltransferase expression and activity, the activity of acetylcholine esterase and potassium-stimulated acetylcholine release in rat hippocampus ${ }^{14-16}$. On the other hand, interaction of cholinergic system and nitric oxide (NO) for manipulation of learning and memory has been suggested ${ }^{17}$.

It has been well-documented that estrogen influences the NO system in both peripheral and nervous tissues. Additionally, estrogen increases endothelial NO synthase (eNOS) and neuronal NO synthase (nNOS) activity and expression and production of NO in tissues including the brain. Therefore, some actions of estradiol are mediated by the $\mathrm{NO}^{18-20}$. It was previously shown that removal of ovaries impairs Morris water maze tasks, which are reversible by L-arginine, the precursor of $\mathrm{NO}^{21}$. It was also shown that the administration of $2 \mathrm{mg} / \mathrm{kg}$ of estradiol valerate improved learning of ovariectomized (OVX), but not sham-operated rats using Morris water maze $\mathrm{e}^{22,23}$.

In the present study, different effects of scopolamine on learning, memory, and NO metabolites levels in hippocampal tissues of OVX and sham oprated rats were investigated.

\section{METHODS}

\section{Animals and drugs}

Thirty-two female Wistar rats (20 weeks-old and weighing $250 \pm 20 \mathrm{~g}$ ) were obtained from the Razi Vaccine and Serum Research Institute (Mashhad, Khorasan province, in Iran). They were housed four per standard cage at $22 \pm 2^{\circ} \mathrm{C}$ in a room with a 12-hour light/dark cycle (light on at 7:00 A.M.), with free access to water and food ad libitum. Rats were given one week to get adapted to the new environment before any procedure was initiated. Animal handling and all related procedures were approved by the Mashhad Medical University Committee on Animal Research. Ketamine and xylazine were purchased from Alfasan Company (Holland). Scopolamine was purchased from Sigma Aldrich (St. Louis MO, USA).

\section{Surgery}

Rats were anesthetized with ketamine (150 mg/kg) and xylazine $(0.1 \mathrm{mg} / \mathrm{kg})$. Anesthesia was confirmed by reduced respiratory rate and no response to gentle pinching of footpad. Ventral incision was made through the skin of the flank of the rat, and ovaries and ovarian fats were removed. Ovaries were isolated by ligation of the most proximal portion of the oviduct before removal. The animals were reversed to their cages to recover from surgery. Two groups of the rats were subjected to sham. The same procedure was almost as ovariectomy, except that the ovaries were not removed ${ }^{21-23}$.

\section{Groups and treatments}

Twelve weeks after surgery, the animals were randomly divided into four groups: Sham; OVX; Sham-Scopolamine (Sham-Sco); and OVX-Scopolamine (OVX-Sco). Animals of Sham-Sco and OVX-Sco Groups received scopolamine ( $2 \mathrm{mg} / \mathrm{kg}$, ip) before testing in Morris water maze. The animals of Sham and OVX Groups were injected by $1 \mathrm{~mL} / \mathrm{kg}$ saline instead of scopolamine.

\section{Morris water maze apparatus and procedures}

A circular black pool (136 cm diameter, $60 \mathrm{~cm}$ high, $30 \mathrm{~cm}$ deep) was filled with water $\left(23\right.$ to $\left.25^{\circ} \mathrm{C}\right)$. A circular platform (10 $\mathrm{cm}$ diameter, $28 \mathrm{~cm}$ high) was placed within the pool and was submerged approximately $2 \mathrm{~cm}$ below the surface of water in the center of the Northwest quadrant. Outside the maze, fixed visual cues were present at various locations around the room (i.e., computer, hardware, and posters). Before every experiment, each rat was daily handled for three days and habituated to the water maze for 30 seconds without a platform. The animals performed four trials on each of the five consecutive days, and each trial began with the rat being placed in the pool and released facing the side wall at one of four positions (the boundaries of the four quadrants, labeled North (N), East (E), South (S), and West (W). Release positions were randomly predetermined. For each trial, the rat was allowed to swim until it found and remained on the platform for 20 seconds. If 60 seconds had passed and the animal had not found the platform, it was guided to the platform by the experimenter and allowed to stay there for 20 seconds. The rat was then removed from the pool, dried and placed in its holding bin for 20 seconds. The time latency to reach the platform and the length of the swimming path were recorded by a video tracking system ${ }^{21-24}$. On the sixth day, the platform was removed, and the animals were allowed to swim for 60 seconds. The time spent in the target quadrant (Q1) was compared between groups. All measurements were performed during the first of the light cycle. 


\section{Biochemical assessment}

After the last session of the Morris water maze test, blood samples were taken from all rats to determine the $\mathrm{NO}$ metabolites NO2 and NO3 (Griess reagent method). The animals were then sacrificed, and hippocampi were removed and submitted to NO metabolite measurements in the tissue. The Griess reaction was adapted to assay nitrates as previously described. Briefly, standard curves for nitrates (Sigma, St.Louis, Missouri, USA) were prepared, and samples (50 $\mu \mathrm{L}$ serum and $100 \mu \mathrm{L}$ tissue suspension) were added to the Griess reagent. Proteins were subsequently precipitated by the addition of $50 \mu \mathrm{L}$ of $10 \%$ trichloroacetic acid (Sigma). The contents were vortex-mixed and centrifuged, and the supernatants were transferred to a 96- well flat-bottomed microplate. Absorbance was read at $520 \mathrm{~nm}$ using a microplate reader, and final values were calculated from standard calibration plots $^{24}$.

\section{Statistical analysis}

All data are expressed as mean \pm standard error of mean (SEM). Swim time latency and length of the traveled path over the five training days were analyzed by repeated analysis of variance (ANOVA) measures. The time spent in the target quadrant (Q1) was compared using one-way ANOVA. Comparison of serum and hippocampal tissues NO metabolites levels was also carried out using one ANOVA. The criterion for statistical significance was $\mathrm{p}<0.05$.

\section{RESULTS}

The time latency and the length of the swimming path over the five training days were significantly higher in OVX Group in comparison with the Sham Group ( $<0.05$ and
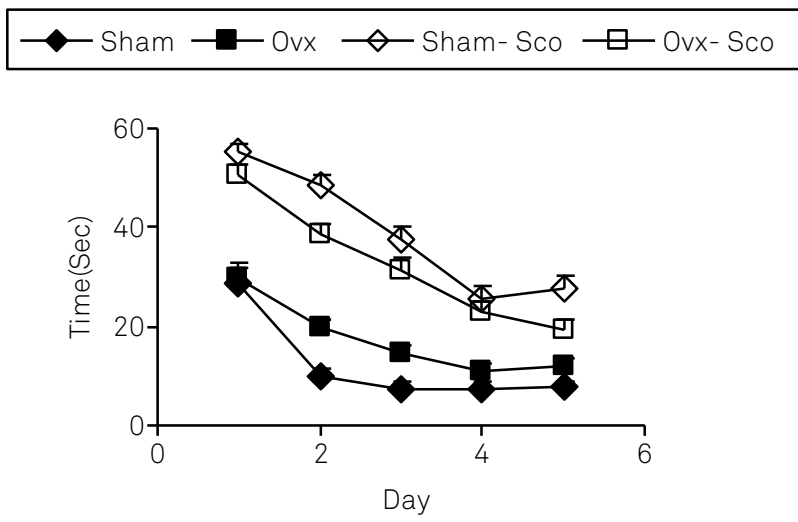

Fig 1. Comparison of swim time latency (seconds) to find the platform among Sham, OVX, Sham-Sco and OVX-Sco Groups. The latency was significantly higher in the OVX Group compared to Sham Group ( $p<0.05)$. The animals of both Sham-Sco and OVX-Sco Groups had significantly higher time latency to reach platform in comparison with those of the Sham and OVX Groups, respectively (both p<0.001). Animals of the Sham-Sco Group had more time to reach the platform in comparison with the OVX-Sco Group ( $p<0.001)$. Data are shown as mean \pm SEM of eight animals per group. $\mathrm{p}<0.01$; Figs 1 and 2, respectively). The animals of both ShamSco and OVX-Sco groups had significantly higher time latency and travelled length to reach platform in comparison with those of Sham and OVX groups, respectively (both $\mathrm{p}<0.001$ ), as seen in Figs 1 and 2. The time latency and the length of the swimming path to reach the platform in the Sham-Sco Group was higher than in the OVX-Sco Group ( $\mathrm{p}<0.001)$. In the probe trial, animals of the Sham-Sco Group spent lower time in Q1 $(\mathrm{p}<0.5)$ and more times in nontarget quadrants $(\mathrm{Q} 3$ and $\mathrm{Q} 4)$, with $\mathrm{p}<0.05$ and $\mathrm{p}<0.001$, in comparison with the Sham Group (Fig 3). Animals of OVX-Sco Group also spent lower time in
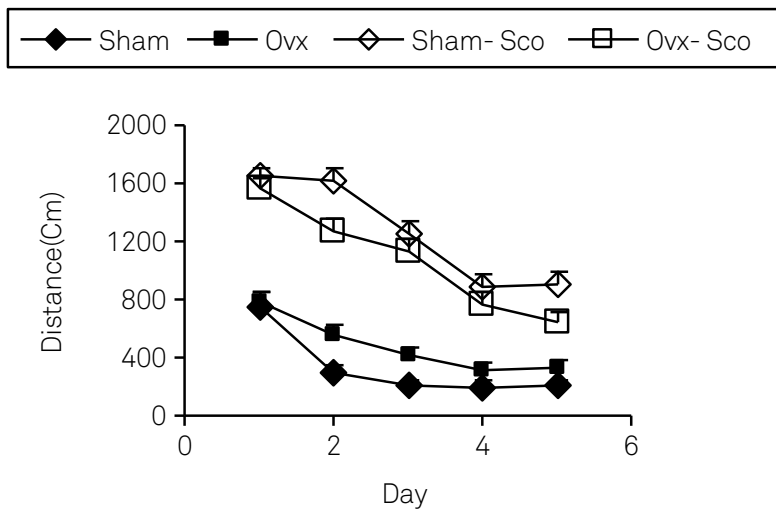

Fig 2. Comparison of the length of the swimming path $(\mathrm{cm})$ to find the platform among Sham, OVX, Sham-Sco and OVX-Sco Groups. The latency was significantly higher in OVX Group compared to Sham Group ( $p<0.01$ ). The animals of both ShamSco and OVX-Sco groups had significantly higher travelled length to reach platform in comparison with those of Sham and OVX groups, respectively ( $p<0.001)$. The animals of ShamSco Group traveled more distant to reach the platform in comparison with the OVX-Sco Group ( $p<0.001)$. Data are shown as mean \pm SEM of eight animals per group.

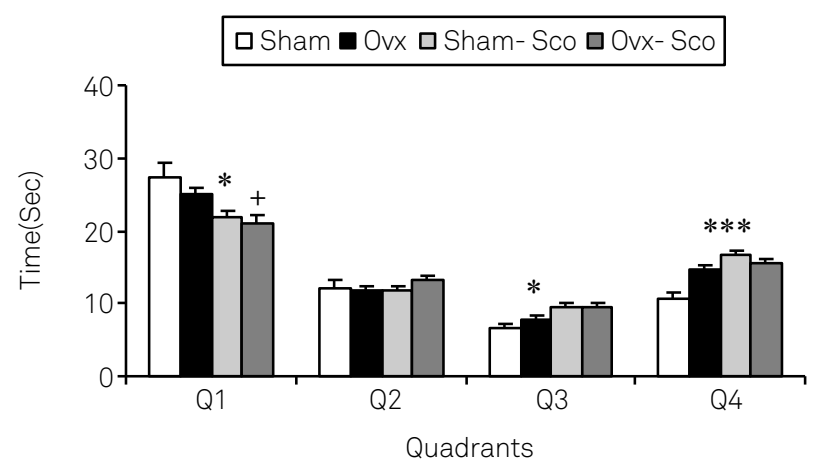

${ }^{*} p<0.05 ;{ }^{* * *} p<0.001$ compared to Sham Group; ${ }^{+} p<0.05$ compared to the OVX Group.

Fig 3. Results of the time (seconds) spent in target quadrant during the probe trial on day 6 (24 hours after the last secession of learning). Data are shown as mean standard error of mean of eight animals per group. The platform was removed, and the time spent in the target quadrant (Q1) was compared between the groups. The time spent in the target quadrant (Q1) by the animals of ShamSco and OVX-Sco groups was significantly lower than Sham and OVX Groups, respectively $(p<0.05)$. 
the Q1 compared to OVX Group $(\mathrm{p}<0.05)$. There were no significant differences in the time spent in the Q2 between the four groups.

NO metabolites concentrations, NO2 or NO3, in the serum of OVX Group, were lower than those of the Sham Group ( $p<0.001$, Fig 4). The serum concentrations of NO2 or NO3 in the Sham-Sco Group were significantly lower than those of the Sham Group ( $p<0.001$, Fig 4). The concentrations of NO metabolites in the hippocampal tissues of the OVX Group were lower than those of the Sham Group ( $p<0.01$, Fig 5$)$. Concentration of NO metabolites in the hippocampi of the Sham-Sco Group was lower than that of the Sham one $(p<0.001$, Fig 5$)$. There was no significant difference between NO2 or NO3 con-

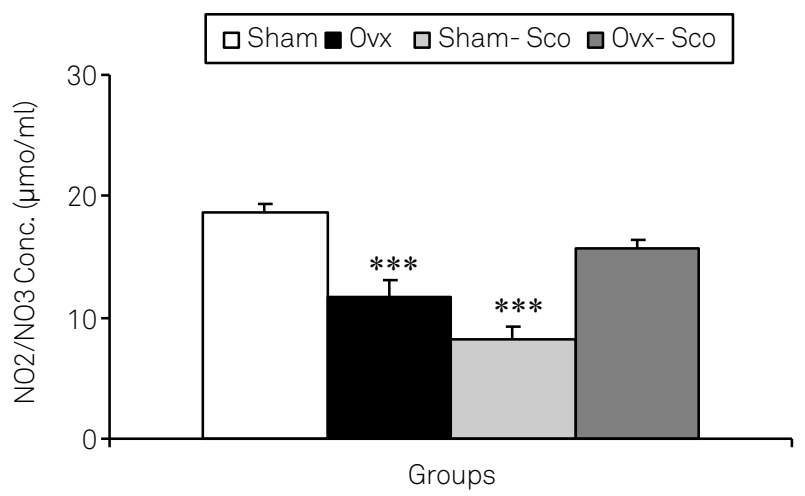

${ }^{* * *} \mathrm{p}<0.001$ compared to the Sham Group.

Fig 4. Comparison of serum NO metabolite levels between four groups. Data are shown as mean \pm SEM of eight animals per group. NO metabolites concentrations in the serum of OVX Group was lower than that of the Sham Group ( $p<0.001)$. The serum concentrations of NO2 or NO3 in the Sham-Sco Group was significantly lower than that of the Sham Group $(p<0.001)$.

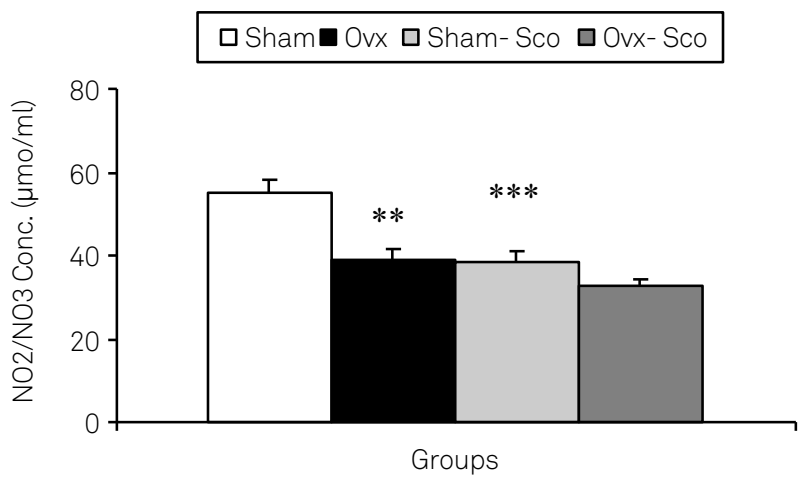

${ }^{* *} p<0.01 ;{ }^{* * *} p<0.001$ compared to the Sham Group.

Fig 5. Comparison of the concentrations of NO metabolites in hippocampal tissues between groups. Data are shown as mean \pm standard error of mean of eight animals per group. The concentrations of $\mathrm{NO}$ metabolites in the hippocampal tissues of the OVX Group were lower than those of the Sham Group ( $p<0.01)$. The concentration of $\mathrm{NO}$ metabolites in the hippocampi of ShamSco Group was lower than that of the Sham one $(p<0.001)$. centrations in the serum and hippocampal tissues of the OVXSco Group compared with the OVX one.

\section{DISCUSSION}

In the present study different effects of scopolamine on learning, memory, and NO metabolites levels in hippocampal tissues of ovariectomized and sham oprated rats were investigated. The ovariectomized rats spent more times and travelled longer distance to reach platform during five days. The results also showed that the sham-operated rats remembered the location of the platform better than ovariectomized rats. Deletion of ovarian hormones impaired learning and memory in Morris water maze. These results were in agreement with the previous studies ${ }^{21-23}$. In contrast with these findings, Herlitz, Thilers and Habib showed that there were no considerable differences in cognitive performance between premenopausal and postmenopausal women ${ }^{25}$. Other researchers have also reported that estrogen has negative effects ${ }^{6}$ or no effect ${ }^{4}$ on learning and memory. It has also been reported that the ovariectomized rats performed better than intact rats at the spatial version of the water maze and, therefore, it seems that the ovarian steroids impair performance on hippocampal-dependent versions of this task ${ }^{26}$. The exact mechanisms by which estrogen regulates the functions of spatial memory have been widely investigated. It has been shown that estrogen affects neurotransmitters, such as glutamate, gamma-Aminobutyric acid (GABA), acetylcholine, and their receptors. Direct or indirect effects of estradiol on hippocampal neurons are also attractive as possible mechanisms ${ }^{27,28}$.

NO contributes in hippocampal dependent learning and memory tasks including spatial memory ${ }^{21-23}$. It has also been suggested that NO mediates the effects of estradiol in many organs $^{29}$. Considering the fact that estradiol affects the release of NO and NOS isoforms activity in the brain, the interaction of estrogen and NO in nervous system functions is conceivable. It has been previously shown that NO contributes in sex hormone-dependent changes of behavior ${ }^{30}$. Previous studies also imply that some functions of estrogen in the central nervous system are related to increased NO production ${ }^{31}$. It was previously shown that L-arginine, the precursor of NO, improves learning and memory impairments in ovariectomized rats. However, it did not have an effect on sham operated ones ${ }^{21}$. It was suggested that regarding the presence of an interaction between estrogen and NO, estrogen deprivation causes to diminish NO in OVX rats and L-arginine could improve spatial memory impairment. Attenuation of the improving effects of estradiol on learning and memory by NO inhibitor L- NAME confirmed this hypothesis ${ }^{23}$. In the present study, learning and memory impairments in ovariectomized rats were accompanied by lower levels of NO2/NO3 in serum and hippocampal tissues in comparison with sham operated rats. These results confirm the involvement of NO in improvement effect of 
estradiol in physiologic conditions. The results obtained from other studies also show that memory impairment is accompanied with low level of $\mathrm{NO}^{32}$. They confirm the role of $\mathrm{NO}$ as a critical mediator in synaptic plasticity, long-term potentiation (LTP), and consolidation of long-term memory. Some researchers also believe that NO production decreases with age and may be involved in Alzheimer's disease ${ }^{32,33}$. Neuronal damage, which occurs in Alzheimer's disease, has been found to result in an impairment of NO synthesis and a decrease in NO containing neurons in the hippocampus ${ }^{34}$. Besides other nurotrasmitters, actycholine has a critical role in learning and memory.

The interaction of cholinergic with NO for manipulation of learning and memory has been suggested. It was shown that co-administration of subthreshold doses of NO inhibitors and muscarinic receptor antagonist, scopolamine, has more deleterious effects on learning and memory of rats in comparison with the adminstration of each of them alone ${ }^{17}$. It has also been reported that NO donors inhibit acetycholinesterase activity and, therefore, it might be suggested that $\mathrm{NO}$ oxide may directly inhibit it ${ }^{34}$. Results of the present study showed for the first time that acetylcholine receptor antagonist, scopolamine, inhibits NO production and the latter may take part in learning and memory impairment due to the administration of scopolamine.

Taking into account that NO interacts with cholinergic system in memory and learning functions and each of NO cholinergic system has interaction with estradiol, we assumed that the effect of scopolamine on learning and memory and NO levels may be different in OVX and sham-operated ones. NO metabolites level in serum and hippocampal tissues of sham operated rats treated by scopolamine was lower than nontreated ones, while there was no significant difference between OVX-Sco Group compared to the OVX Group. It seems that scopolamine differently affects $\mathrm{NO}$ in the presence and absence of ovarian hormones.

In conclusion, NO oxide may be involved in deletrious effects of scopolamine on learning and memory. Our results also indicate that the effect of scopolamine on $\mathrm{NO}$ metabolites levels is different in the presence or absence of ovarian hormones, however further investigations need to be carried out.

\section{References}

1. Hojo Y, Hattori TA, Enami T, et al. Adult male rat hippocampus synthesizes estradiol from pregnenolone by cytochromes P45017alpha and P450 aromatase localized in neurons. Proc Natl Acad Sci U S A 2004;101:865-870.

2. Kretz O, Fester L, Wehrenberg U, et al. Hippocampal synapses depend on hippocampal estrogen synthesis. J Neurosci 2004;24:5913-5921.

3. Bimonte HA, Denenberg VH. Estradiol facilitates performance as working memory load increases. Psychoneuroendocrinology 1999;24:161-173.

4. Healy SD, Braham SR, Braithwaite VA. Spatial working memory in rats: no differences between the sexes. Proceedings Of The Royal Society Of London Series B: Biological Sciences 1999;266:2303-2308.

5. Sherwin BB. Estrogen and memory in women: how can we reconcile the findings? Horm Behav 2005;47:371-375.

6. Chesler EJ, Juraska JM. Acute administration of estrogen and progesterone impairs the acquisition of the spatial Morris water maze in ovariectomized rats. Hormones Behav 2000;38:234-242.

7. Kritzer MF, Kohama SG. Ovarian hormones differentially influence immunoreactivity for dopamine beta- hydroxylase, choline acetyltransferase, and serotonin in the dorsolateral prefrontal cortex of adult rhesus monkeys. J Comp Neurol 1999;409:438-451.

8. Adler A, Vescovo P, Robinson JK, Kritzer MF. Gonadectomy in adult life increases tyrosine hydroxylase immunoreactivity in the prefrontal cortex and decreases open field activity in male rats. Neuroscience 1999;89:939-954.

9. Bethus I, Tse D, Morris RG. Dopamine and memory: modulation of the persistence of memory for novel hippocampal NMDA receptordependent paired associates. J Neurosci 2010;30:1610-1618.

10. Sagrillo CA, Selmanoff M. Castration decreases single cell levels of mRNA encoding glutamic acid decarboxylase in the diagonal band of broca and the sexually dimorphic nucleus of the preoptic area. $J$ Neuroendocrinol 1997;9:699-706.

11. Sumner BE, Fink G. Testosterone as well as estrogen increases serotonin2A receptor $\mathrm{mRNA}$ and binding site densities in the male rat brain. Brain Res Mol Brain Res 1998;59:205-214.
12. Perry EK, Tomlinson BE, Blessed G, Bergmann K, Gibson PH, Perry $\mathrm{RH}$. Correlation of cholinergic abnormalities with senile plaques and mental test scores in senile dementia. Br Med J 1978;2:1457-1459.

13. Fine A, Dunnett SB, Bjorklund A, Iversen SD. Cholinergic ventral forebrain grafts into the neocortex improve passive avoidance memory in a rat model of Alzheimer disease. Proc Natl Acad Sci U S A 1985;82:5227-5230.

14. McMillan PJ, Singer CA, Dorsa DM. The effects of ovariectomy and estrogen replacement on trkA and choline acetyltransferase mRNA expression in the basal forebrain of the adult female Sprague-Dawley rat.J Neurosci 1996;16:1860-1865.

15. Luine VN, McEwen BS. Sex differences in cholinergic enzymes of diagonal band nuclei in the rat preoptic area. Neuroendocrinology 1983;36:475-482

16. Gibbs RB. Effects of estrogen on basal forebrain cholinergic neurons vary as a function of dose and duration of treatment. Brain Res 1997;757:10-16.

17. Pistell PJ, Daffin LW Jr., Nelson CM, et al. Combined administration of subthreshold doses of the nitric oxide inhibitor, nitro-L-arginine, and muscarinic receptor antagonist, scopolamine, impairs complex maze learning in rats. Behav Pharmacol 2007;18:801-805.

18. Stefano GB, Prevot V, Beauvillain JC, et al. Cell-surface estrogen receptors mediate calcium-dependent nitric oxide release in human endothelia. Circulation 2000;101:1594-1597.

19. Russell KS, Haynes MP, Caulin-Glaser T, Rosneck J, Sessa WC, Bender JR. Estrogen stimulates heat shock protein 90 binding to endothelial nitric oxide synthase in human vascular endothelial cells. Effects on calcium sensitivity and NO release.J Biol Chem 2000;275:5026-5030.

20. Russell ME, Wallace AF, Wyner LR, Newell JB, Karnovsky MJ. Upregulation and modulation of inducible nitric oxide synthase in rat cardiac allografts with chronic rejection and transplant arteriosclerosis. Circulation 1995;92:457-464.

21. Saffarzadeh F, Eslamizade MJ, Nemati HAK, Hadjzadeh MA, Khazaei $M$, Hosseini M. The effect of L-arginine on Morris water maze tasks of ovariectomized rats. Acta Physiol Hung 2010;97:216-223. 
22. Hosseini M, Headari R, Oryan S, Hadjzadeh MA, Saffarzadeh F, Khazaei M. The effect of chronic administration of L-arginine on the learning and memory of estradiol-treated ovariectomized rats tested in the morris water maze. Clinics (São Paulo) 2010;65:803-807.

23. Azizi-Malekabadi H, Hosseini M, Saffarzadeh F, Karami R, Khodabandehloo F. Chronic treatment with the nitric oxide synthase inhibitor, L-NAME, attenuates estradiol-mediated improvement of learning and memory in ovariectomized rats. Clinics (São Paulo) 2011;66:673-679.

24. Hosseini M, Dastghaib SS, Rafatpanah H, Hadjzadeh MA, Nahrevanian $H$, Farrokhi I. Nitric oxide contributes to learning and memory deficits observed in hypothyroid rats during neonatal and juvenile growth. Clinics (São Paulo) 2010;65:1175-1181.

25. Herlitz A, Thilers P, Habib R. Endogenous estrogen is not associated with cognitive performance before, during, or after menopause. Menopause 2007;14:425-431.

26. Daniel JM, Roberts SL, Dohanich GP. Effects of ovarian hormones and environment on radial maze and water maze performance of female rats. Physiol Behav 1999;66:11-20.

27. Gazzaley AH, Weiland NG, McEwen BS, Morrison JH. Differential regulation of NMDAR1 $\mathrm{mRNA}$ and protein by estradiol in the rat hippocampus.J Neurosci 1996;16:6830-6838.

28. Daniel JM, Dohanich GP.Acetylcholine mediates the estrogen-induced increase in NMDA receptor binding in CA1 of the hippocampus and the associated improvement in working memory. $J$ Neurosci 2001;21:6949-6956.

29. Gotti S, Martini M, Viglietti-Panzica C, Miceli D, Panzica G. Effects of estrous cycle and xenoestrogens expositions on mice nitric oxide producing system. Ital J Anat Embryol 2010;115:103-108.

30. Hosseini M, Sadeghnia HR, Salehabadi S, Alavi H, Gorji A. The effect of L-arginine and L-NAME on pentylenetetrazole induced seizures in ovariectomized rats, an in vivo study. Seizure 2009;18:695-698.

31. Lopez-Jaramillo P, Teran E. Improvement in functions of the central nervous system by estrogen replacement therapy might be related with an increased nitric oxide production. Endothelium 1999;6:263266.

32. Kuiper MA, Visser JJ, Bergmans PL, Scheltens P, Wolters EC. Decreased cerebrospinal fluid nitrate levels in Parkinson's disease, Alzheimer's disease and multiple system atrophy patients. J Neurol Sci 1994;121:46-49.

33. Reckelhoff JF, Kellum JA, Blanchard EJ, Bacon EE, Wesley AJ, Kruckeberg WC. Changes in nitric oxide precursor, L-arginine, and metabolites, nitrate and nitrite, with aging. Life Sci 1994;55: 1895-1902.

34. Udayabanu M, Kumaran D, Nair RU, et al. Nitric oxide associated with iNOS expression inhibits acetylcholinesterase activity and induces memory impairment during acute hypobaric hypoxia. Brain Res 2008;1230:138-149. 